Supporting Information

\title{
Optimized Thermoelectric Performance of Carbon Nanoparticle- Carbon Nanotube Heterostructures by Tuning Interface Barrier Energy
}

Woomin Park, Hyeonseok Hwang, Sohee Kim, Sungbin Park, and Kwang-Suk Jang*

Department of Applied Chemistry (Major in Bionano Convergence), Hanyang University, Ansan, Gyeonggi-do 15588, Republic of Korea.E-mail: kjang@hanyang.ac.kr 


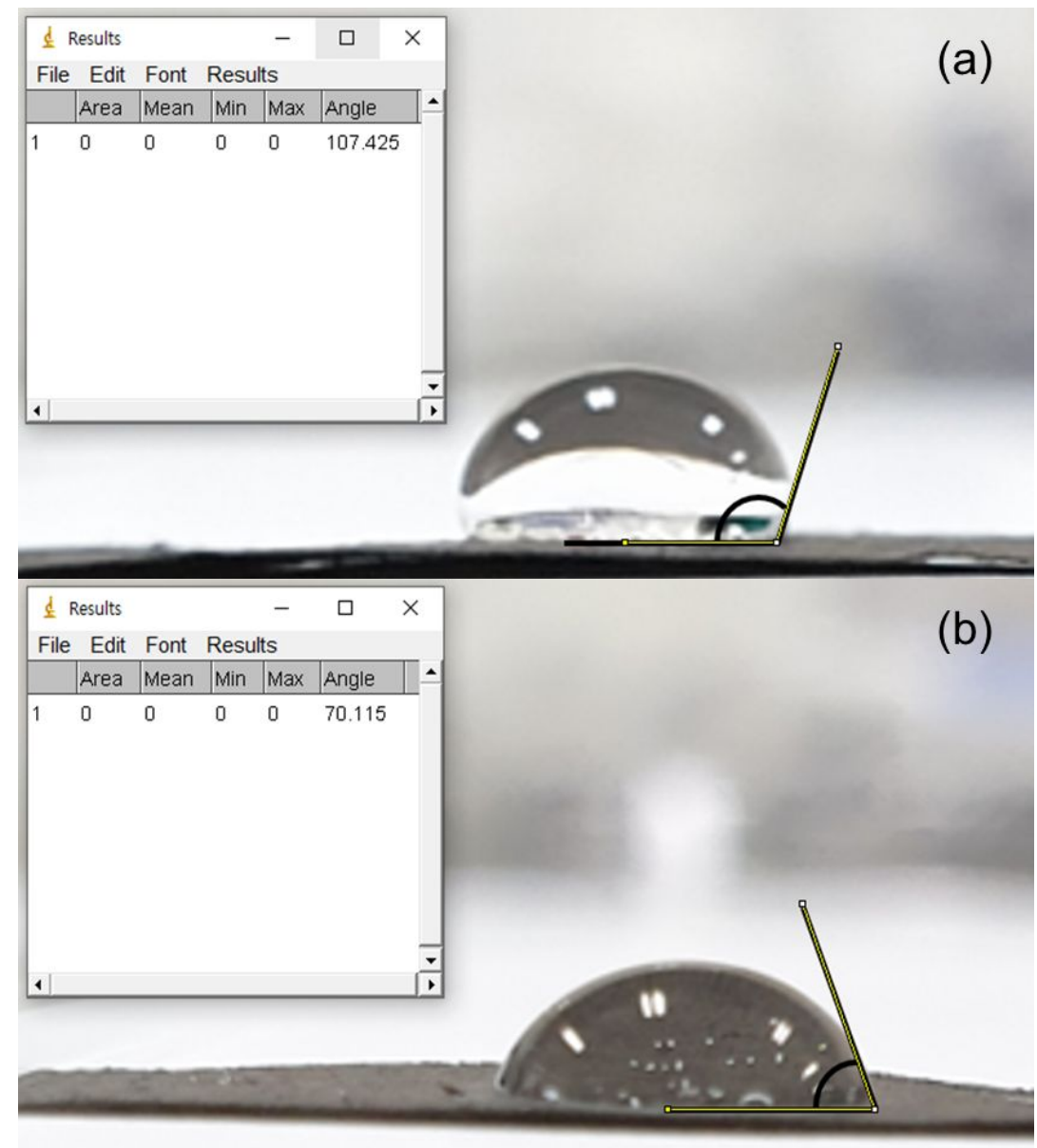

Figure S1. Contact angles of the as-prepared and $800^{\circ} \mathrm{C}$-pyrolyzed CNT films. 


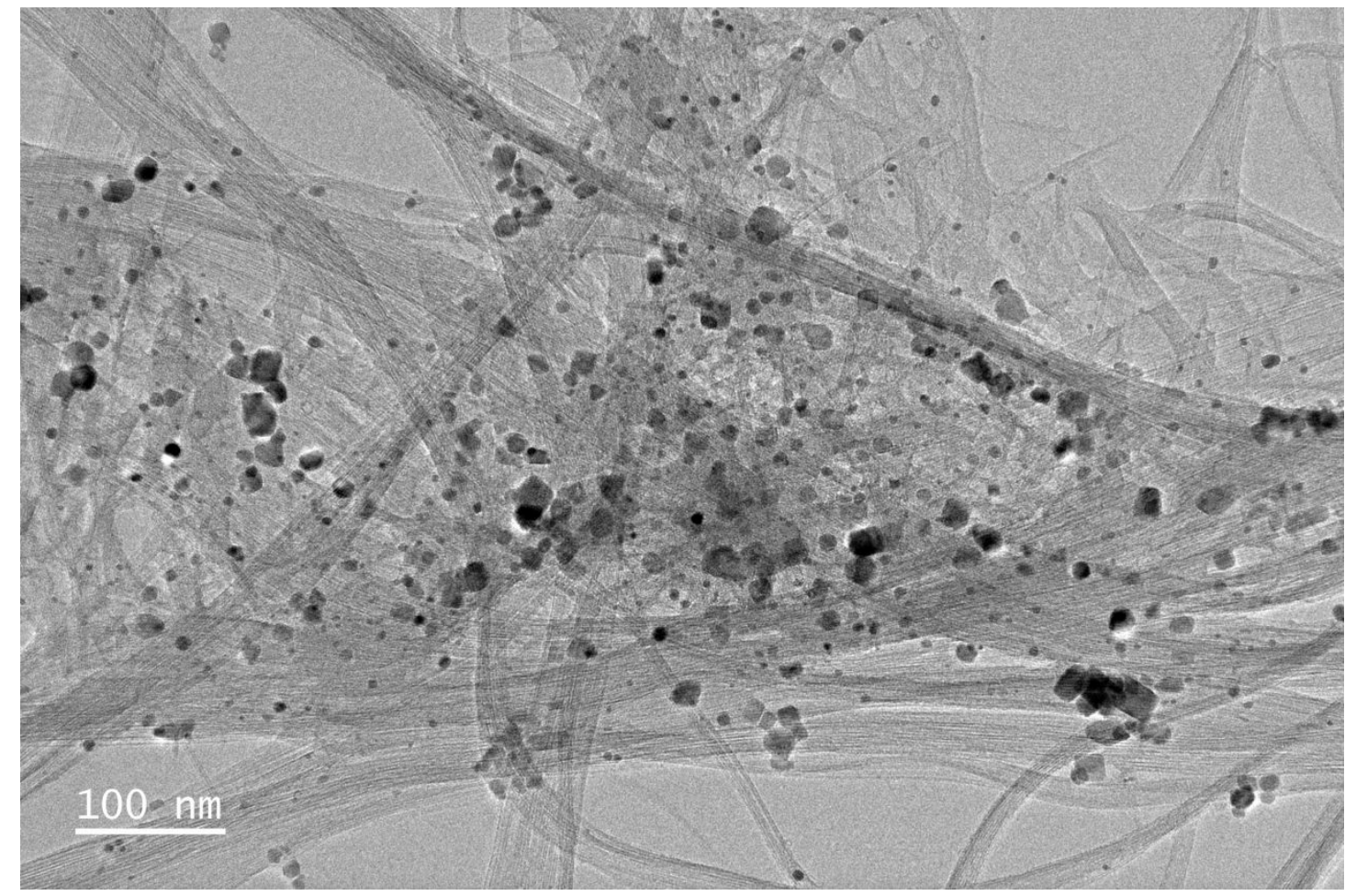

Figure S2. TEM image of the $800^{\circ} \mathrm{C}$-pyrolyzed glucose-CNT film with a glucose content of $70 \mathrm{wt} \%$. 

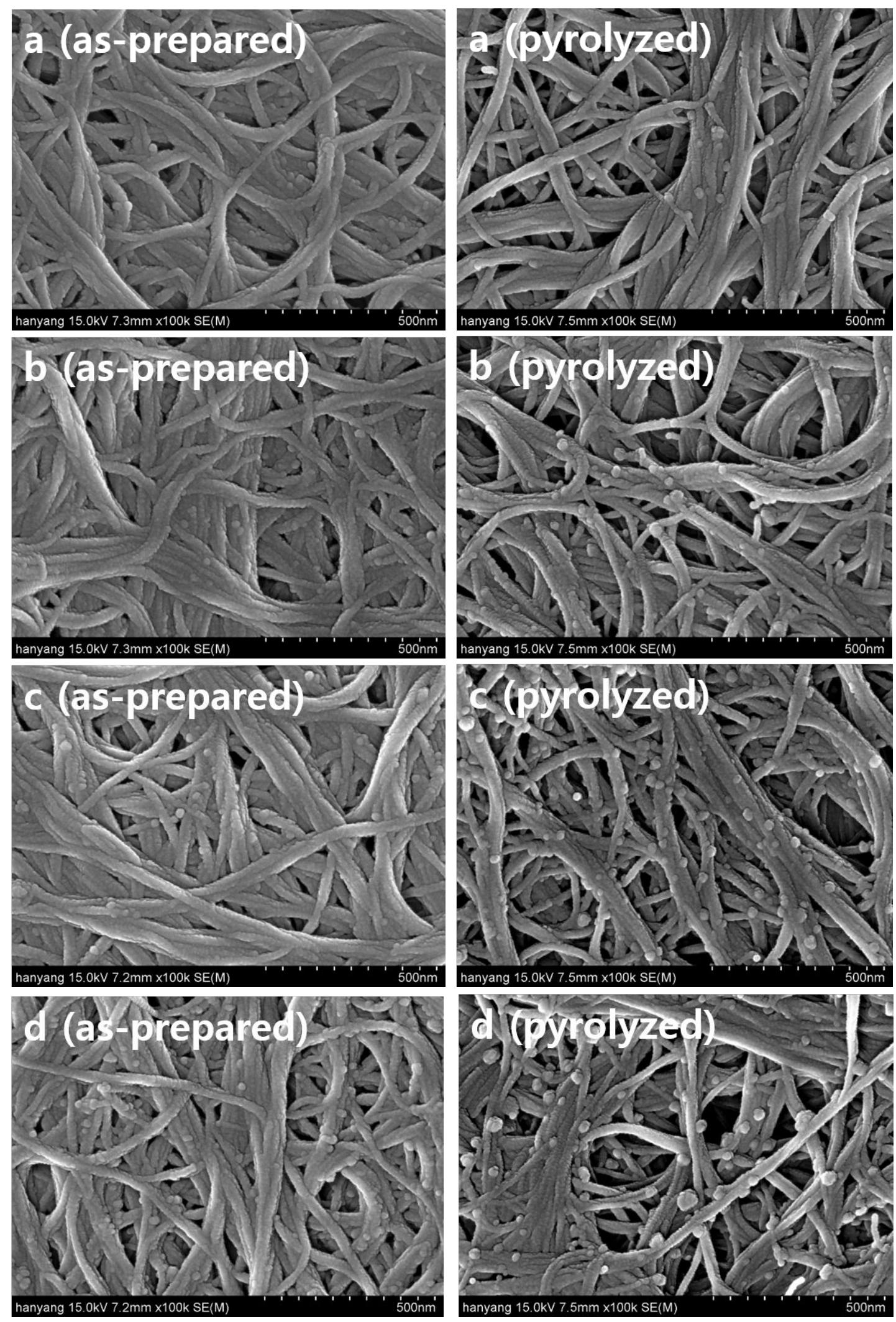

Figure S3. SEM images of the as-prepared and pyrolyzed glucose-CNT films with glucose contents of (a) 10, (b) 30, (c) 50, and (d) $70 \mathrm{wt} \%$. 

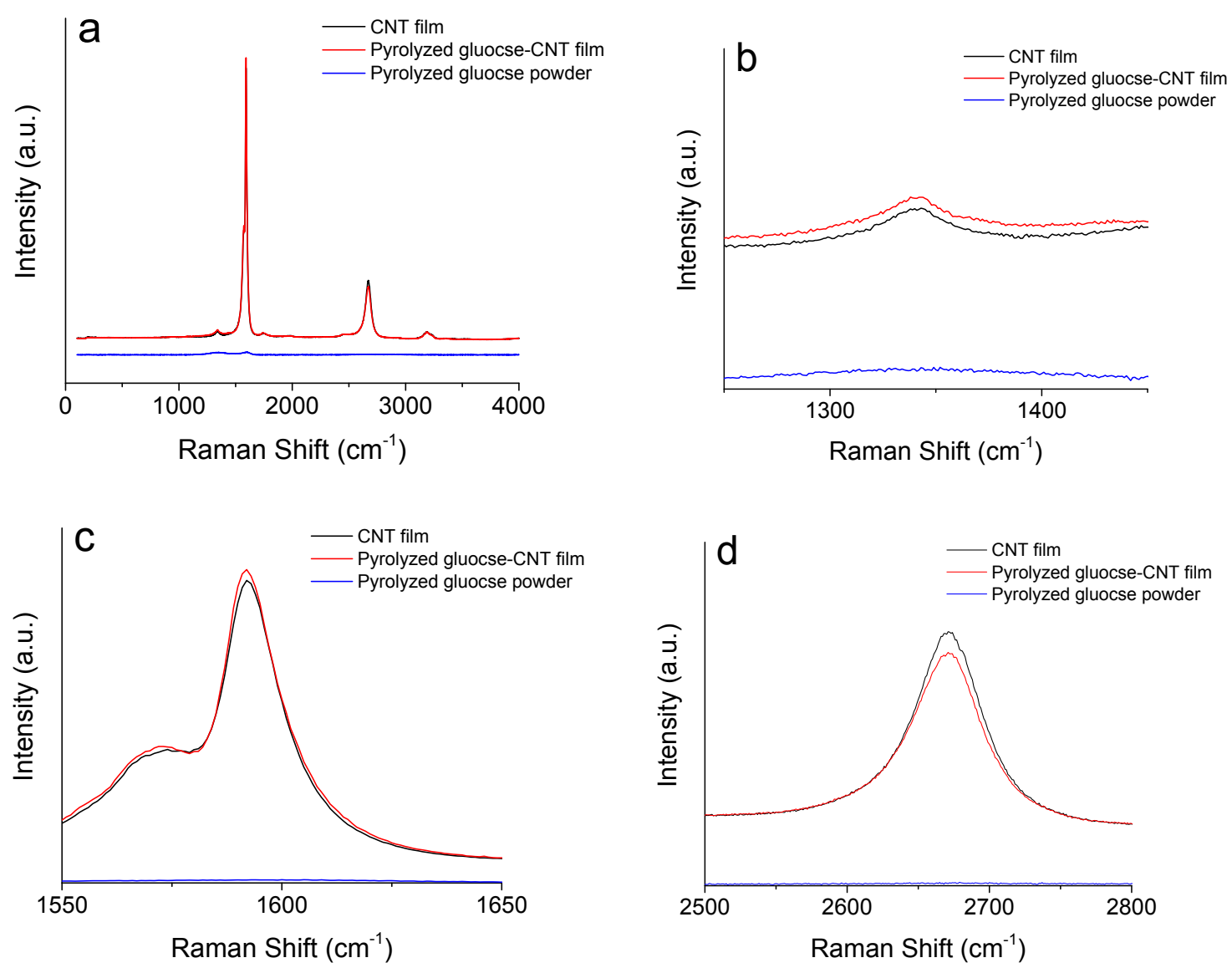

Figure S4. (a) Full range, (b) D band, (c) G band, and (d) 2D band Raman spectra of the CNT film, $800{ }^{\circ} \mathrm{C}$-pyrolyzed glucose-CNT films with a glucose content of $70 \mathrm{wt} \%$, and $800{ }^{\circ} \mathrm{C}$-pyrolyzed glucose powders. 


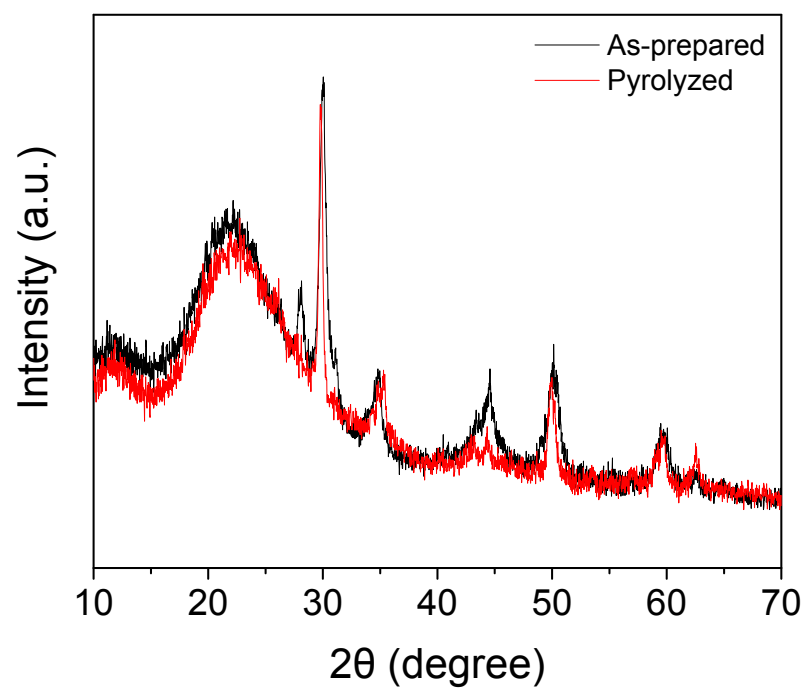

Figure S5. X-ray diffraction patterns of the as-prepared and $800{ }^{\circ} \mathrm{C}$-pyrolyzed glucose-CNT films with a glucose content of $70 \mathrm{wt} \%$. 

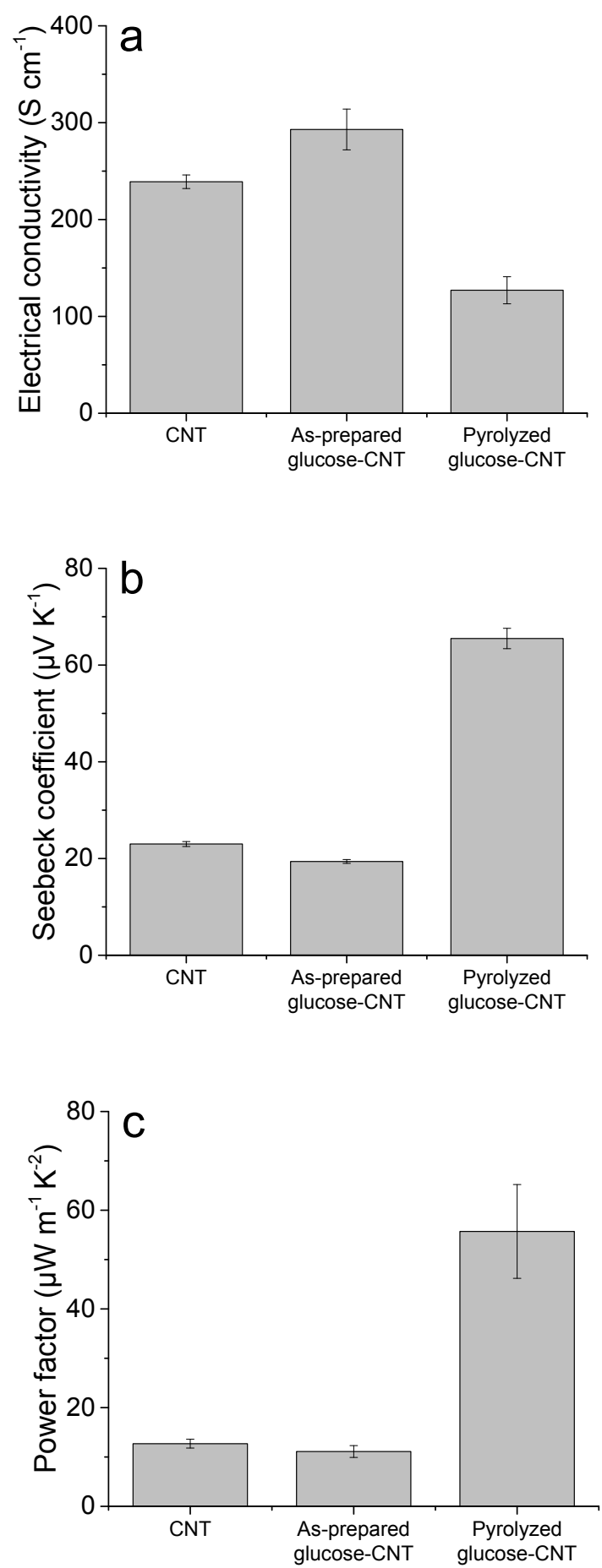

Figure S6. (a) $\sigma$, (b) $S$, and (c) $P F$ values of the CNT films, and as-prepared and $800{ }^{\circ} \mathrm{C}$-pyrolyzed glucose-CNT films with a glucose content of $70 \mathrm{wt} \%$, prepared by using HiPco single-walled CNTs. The super purified HiPco single-walled CNTs (purity: $\geq 95 \%$ ) were purchased from Unidym. The CNTs have diameters of $0.8-1.2 \mathrm{~nm}$ and lengths of $0.1-1 \mu \mathrm{m}$. 

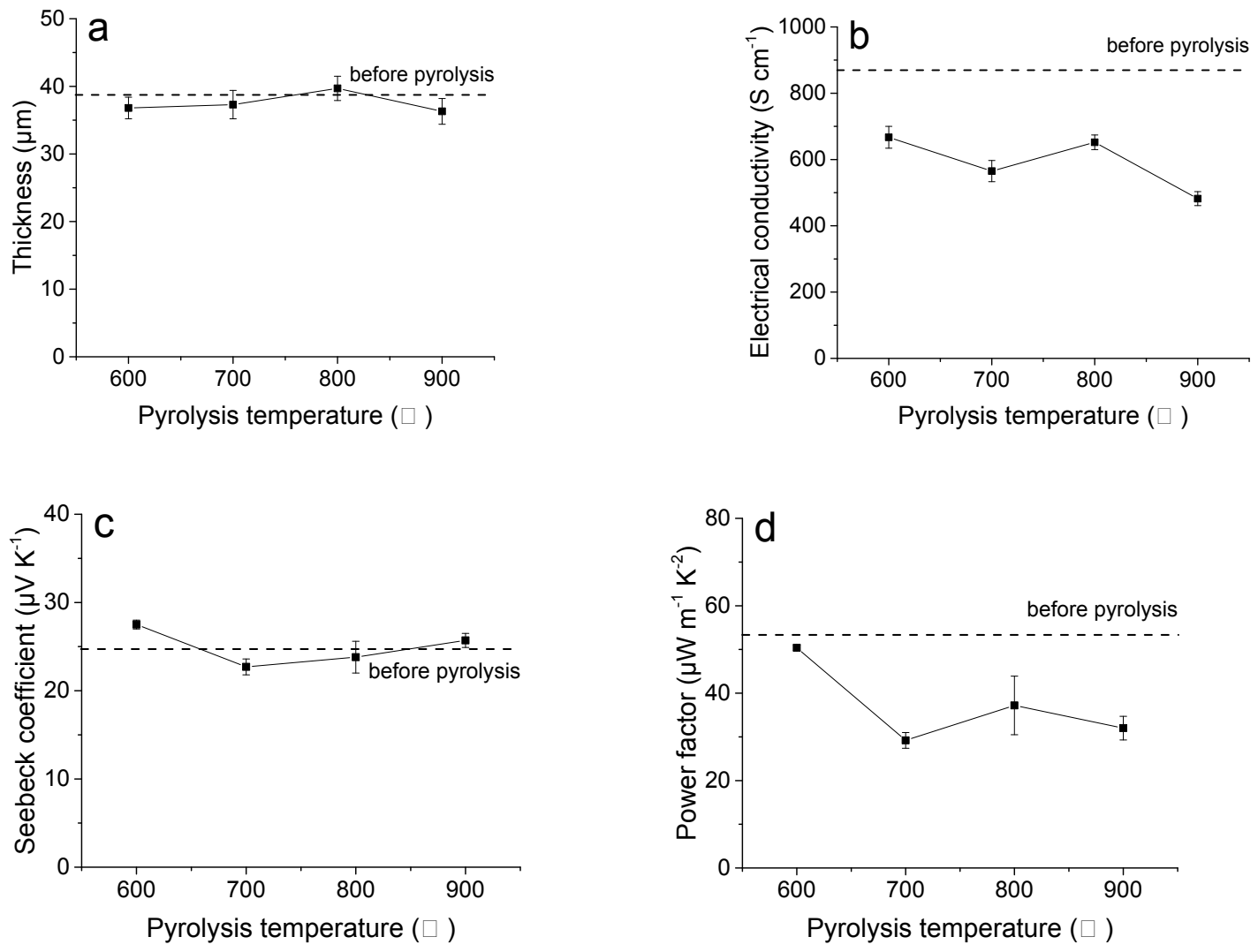

Figure S7. (a) Thickness, (b) $\sigma$, (c) $S$, and (d) $P F$ values of pyrolyzed CNT films as a function of the pyrolysis temperature. 

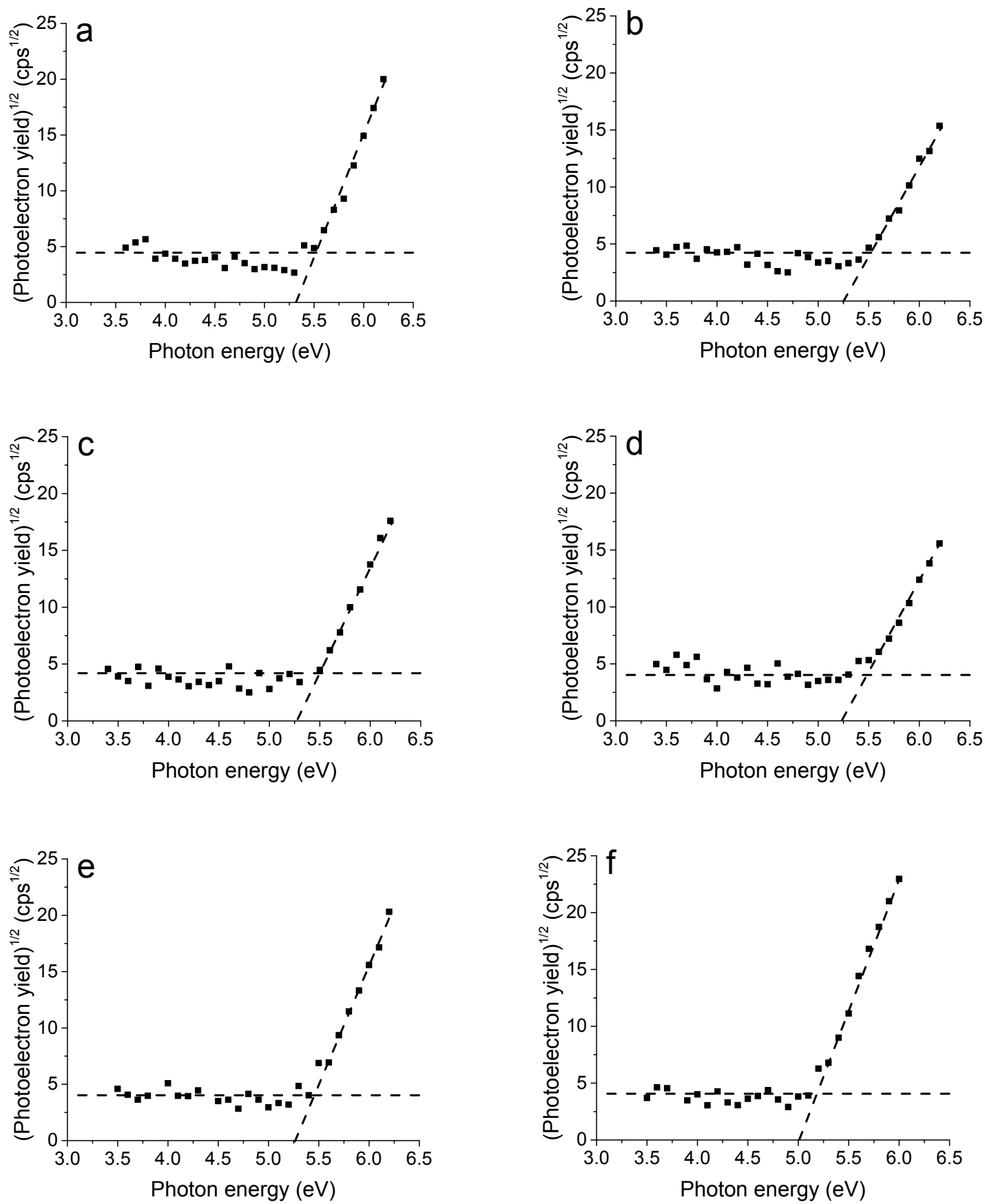

Figure S8. Square roots of the photoelectron emission intensities as a function of the irradiated photon energy for the pyrolyzed glucose-urea mixtures with urea contents of (a) 0 , (b) 1 , (c) 2.5, (d) 10 , (e) 35 , and (f) $65 \mathrm{wt} \%$. 


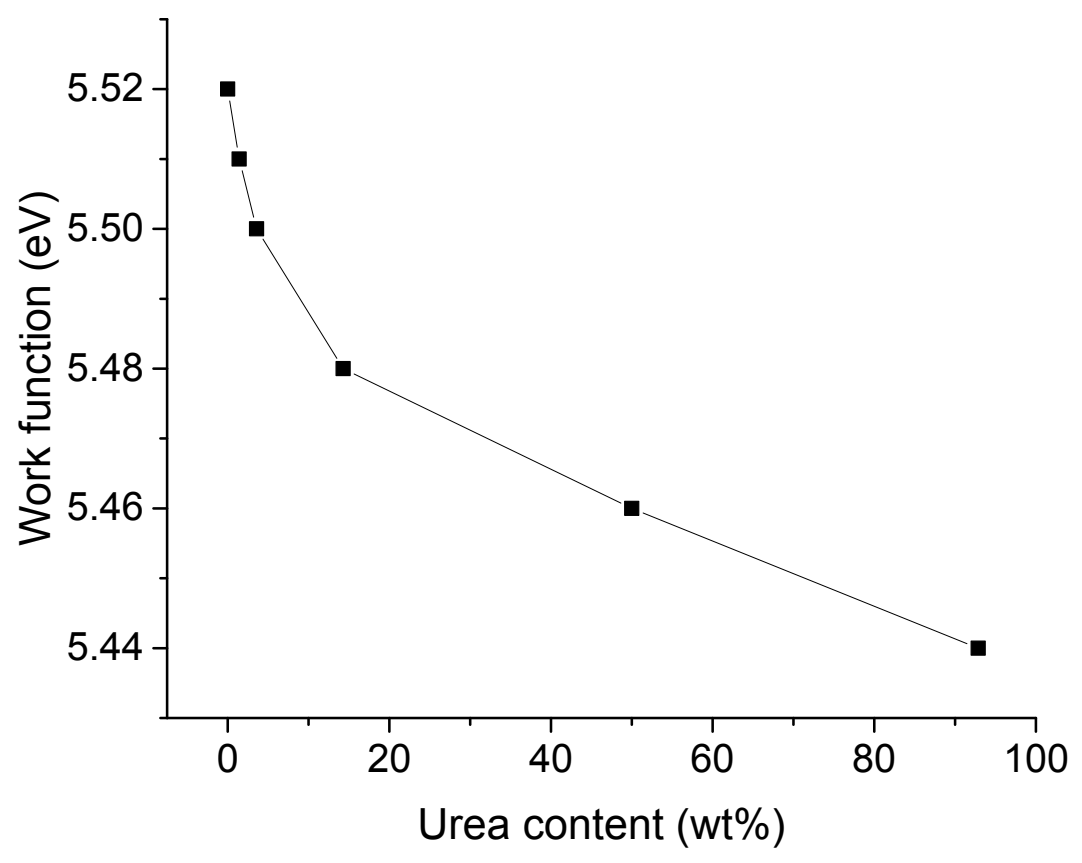

Figure S9. Work functions of pyrolyzed glucose-urea mixtures as a function of urea content. 

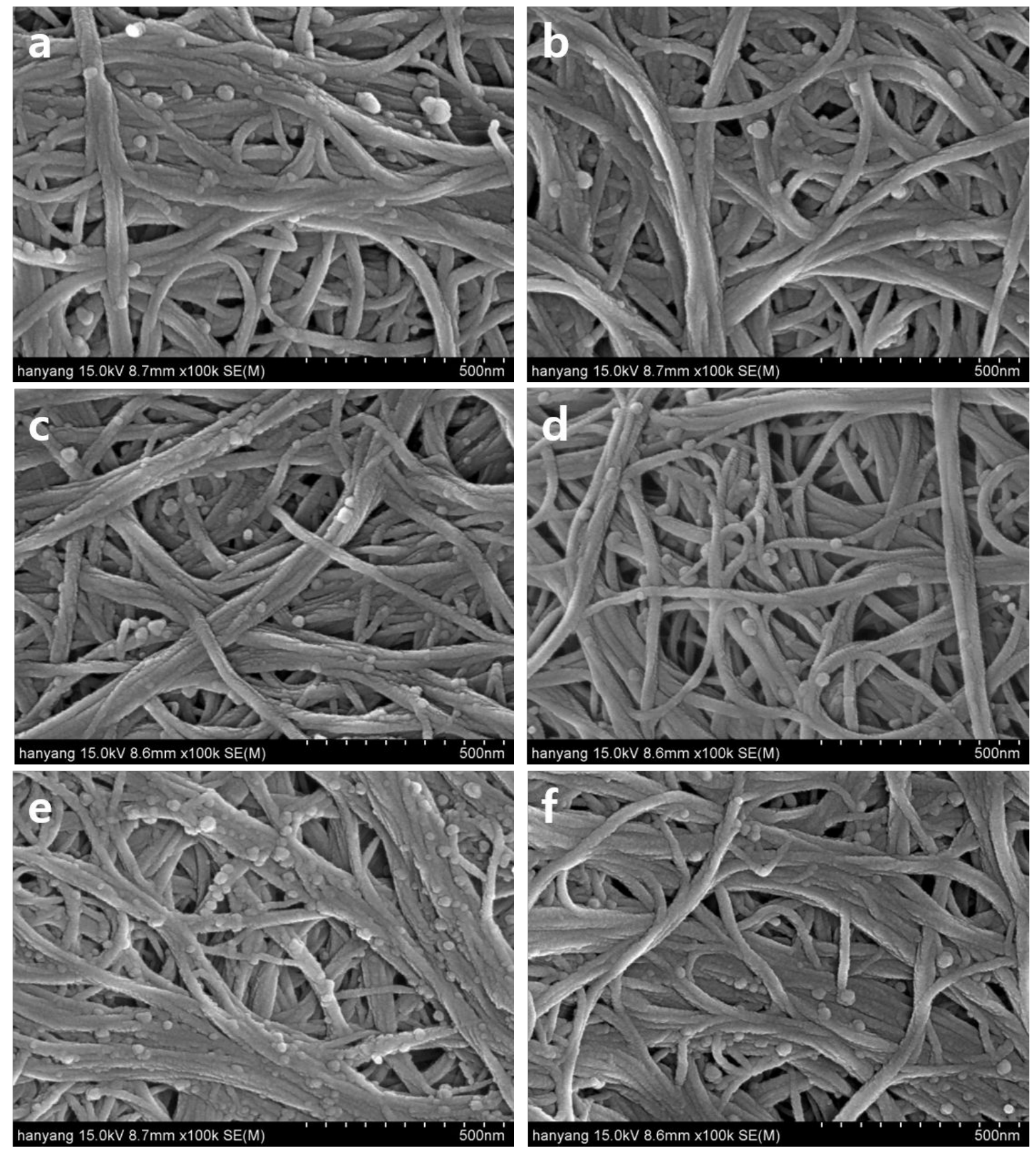

Figure S10. SEM images of the pyrolyzed glucose-urea-CNT films with urea contents of (a) 1, (b) 2.5, (c) 5 , (d) 10 , (e) 35 , and (f) $65 \mathrm{wt} \%$. 

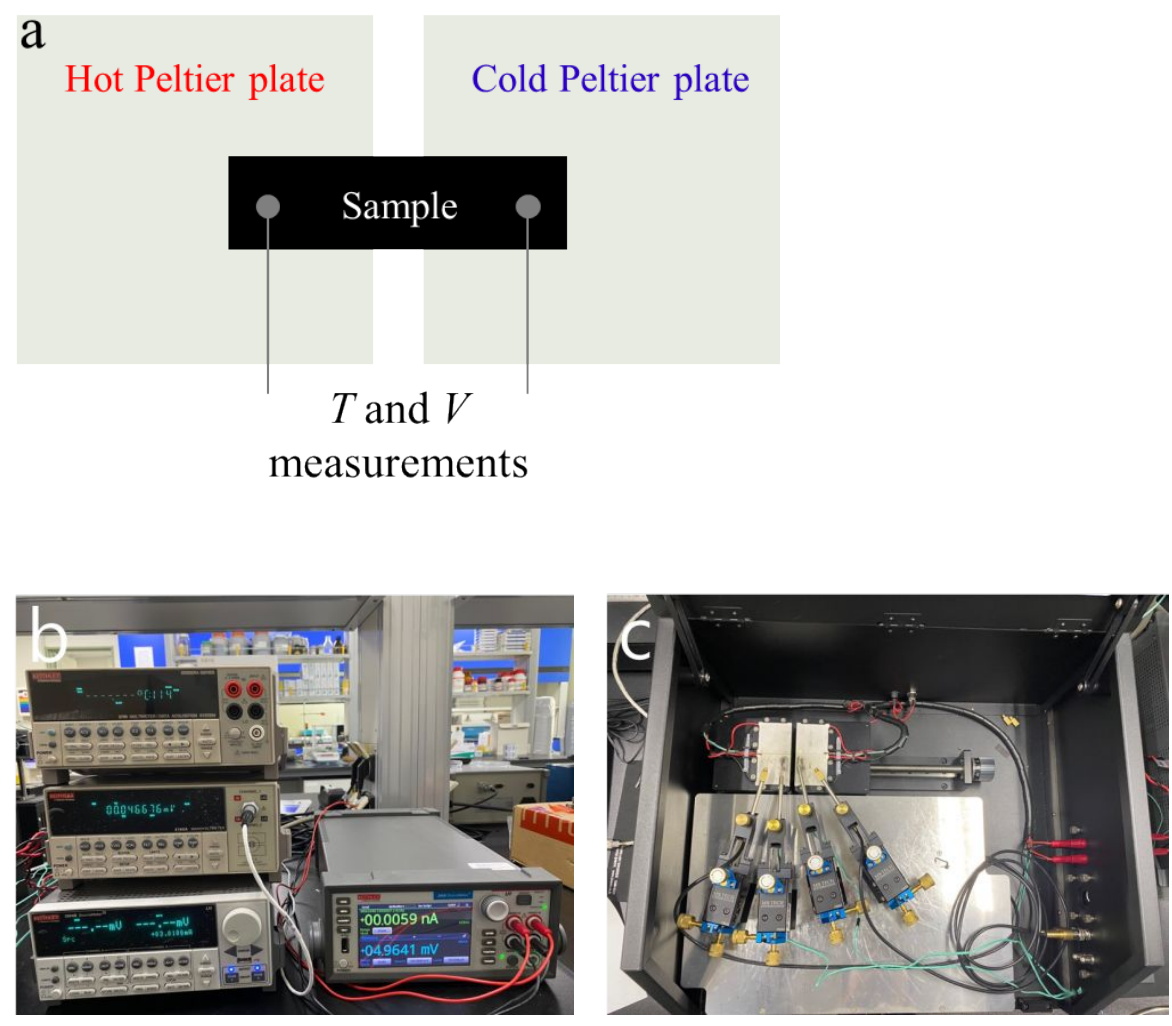

Figure S11. Scheme for the $\Delta T$ and $\Delta V$ measurement of the thermoelectric film. Photographs of (b) the temperature control and electrical measurement part and (c) the measurement stage part. The temperature difference was controlled using Peltier devices, which were controlled by a Keithley 2200 power source and a Keithley 2460 source meter. 


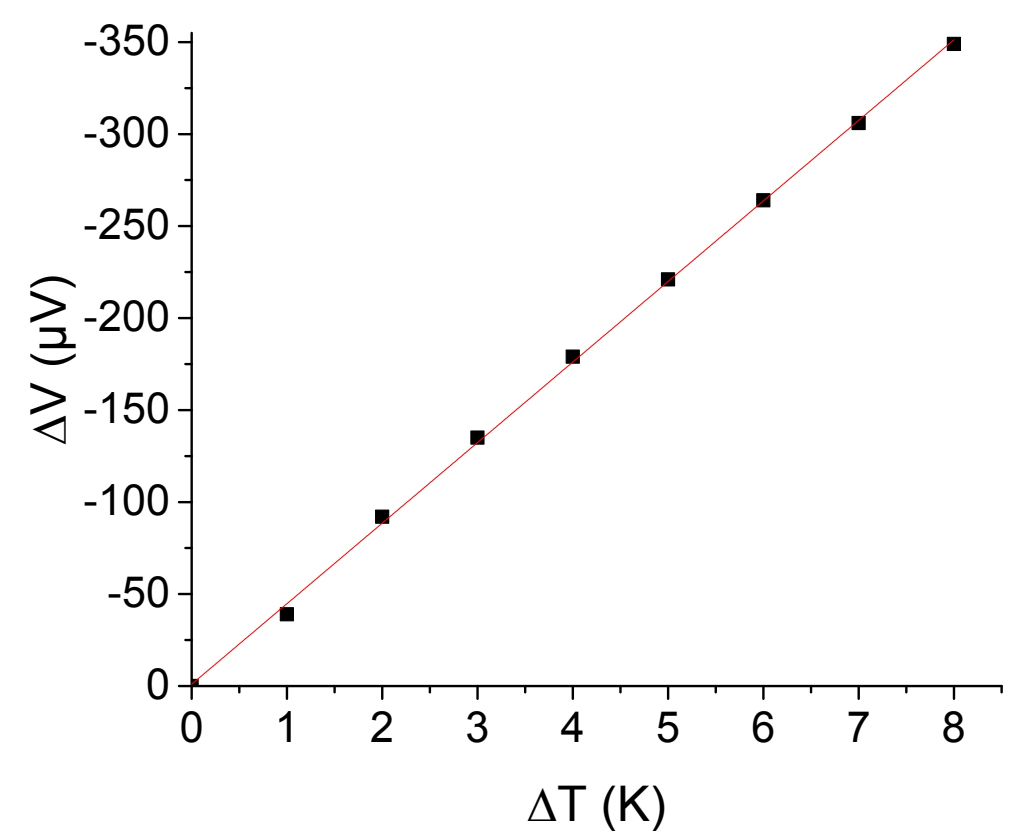

Figure S12. The $\Delta V-\Delta T$ curve of the constantan at room temperature. The measured Seebeck coefficient of the constantan at room temperature is $-39.0 \mu \mathrm{V} \mathrm{K}^{-1}$, which is well correspond to the reported value..$^{\text {s1 }}$

(S1) Guan, A.; Wang, H.; Jin, H.; Chu, W.; Guo, Y.; Lu, G. An Experimental Apparatus for Simultaneously Measuring Seebeck Coefficient and Electrical Resistivity from $100 \mathrm{~K}$ to $600 \mathrm{~K}$. Rev. Sci. Instrum. 2013, 84, 043903. 